\title{
Linear electro-optic effect due to the built-in electric field in InGaN/GaN quantum wells
}

\author{
Hongtao Jiang ${ }^{\text {a) }}$ and Jasprit Singh \\ Department of Electrical Engineering and Computer Science, The University of Michigan, Ann Arbor, \\ Michigan 48105
}

(Received 20 April 1999; accepted for publication 6 August 1999)

A strong piezoelectric effect and large lattice mismatch allow one to incorporate high built-in electric fields in InGaN/GaN quantum wells. This letter examines the implications of these fields on the absorption spectra and refractive index changes induced by an external perpendicular electric field. We find that InGaN/GaN quantum wells show linear electro-optic effect due to quantum confined Stark effect. Our results suggest application of InGaN/GaN quantum wells in Mach-Zehnder type modulators and in electroabsorption modulators in the blue light region.

(C) 1999 American Institute of Physics. [S0003-6951(99)05439-X]

Optical modulators are attractive devices for low chirp modulation of lasers, optical switches, and programmable transparencies. In quantum well systems, quantum confined Stark effect, (QCSE) allows one to exploit both electroabsorption modulators and modulators using the electro-optic effect. Both experimental and theoretical work has been reported for the important 1.5 and $1.33 \mu \mathrm{m}$ InGaAsP/InP systems. ${ }^{1-4}$ Because of the symmetry of these square quantum wells, all effects associated with QCSE are of even order of the electric field. This limits its application to the case of relatively large external voltage bias. It is well known that for triangular quantum wells and for wells with a built-in electric field, it is possible to have linear electro-optic effect.

Recent advances in GaN based heterostructures have led to the demonstration of light emitting diodes and laser diodes. ${ }^{5,6}$ The interest in these systems arises due to possible applications in the short wavelength regime. While there have been studies of light emission properties of nitride based quantum wells, there has been no work examining how the electric field modulates the optical properties of these materials. The study of electric field modulation properties of InGaN/GaN quantum well is of practical interest for electroabsorption and electric-optical effect modulators. In the InGaN quantum wells, there exists a strong piezoelectric field due to the lattice mismatch between $\mathrm{InGaN}$ well region and GaN barrier layer. The magnitude of this field can easily approach $\mathrm{MV} / \mathrm{cm}$. It is important to know what the implications of this field are. In this letter, we study the modulation properties of InGaN quantum wells. In addition to the calculation of the electro-optic properties, we find the existence of first order electro-optic effect.

The polarization of the strained layer is the sum of the spontaneous polarization and polarization due to the piezoelectric effect. The spontaneous polarization constants for $\mathrm{InN}$ and $\mathrm{GaN}$ are very close. ${ }^{7}$ As a result, we expect that spontaneous polarization will have a negligible effect on the internal field in InGaN/GaN quantum wells. On the other hand, there is a large lattice mismatch between $\mathrm{GaN}$ and $\mathrm{InN}$ and the piezoelectric constants are quite large, the piezoelec-

${ }^{\text {a)} E l e c t r o n i c ~ m a i l: ~ j i a n g h t @ e n g . u m i c h . e d u ~}$ tric field is expected to be large for the strained layer. The piezoelectric field $F_{\mathrm{pz}}$ for the biaxial strained layer is given by

$$
F_{\mathrm{pz}}=-\frac{1}{\epsilon_{r} \epsilon_{0}}\left[e_{33} \epsilon_{z z}+e_{31}\left(\epsilon_{x x}+\epsilon_{y y}\right)\right],
$$

where $\boldsymbol{\epsilon}_{x x}=\boldsymbol{\epsilon}_{y y}=\left(a-a_{0}\right) / a_{0}, \boldsymbol{\epsilon}_{z z}=-2 \epsilon_{x x} c_{13} / c_{33}, a_{0}$ is the unstrained lattice constant and $a$ is the lattice constant at strained case, $c_{13}$ and $c_{33}$ are elastic constants, $\epsilon_{r}$ is relative dielectric constant and it is around 10.18 for $\mathrm{In}_{0.2} \mathrm{Ga}_{0.8} \mathrm{~N}$, and $\epsilon_{0}$ is the permeability of vacuum. There is some variation in the values of piezoelectric constants reported by various references. $^{7-13}$ For the calculations reported here, we use $e_{33}-3.26 e_{31}=0.77 \mathrm{C} / \mathrm{m}^{2}$ with $c_{13}=109 \mathrm{GPa}, c_{33}=355 \mathrm{GPa}$. The values used above give a piezoelectric field of 1.1 $\mathrm{MV} / \mathrm{cm}$ for $\mathrm{In}_{0.2} \mathrm{Ga}_{0.8} \mathrm{~N} / \mathrm{GaN}$. This value falls in the range of values from optical measurements of the InGaN/GaN quantum wells and heterostructures. ${ }^{9-12}$

To calculate the optical properties in $\mathrm{InGaN} / \mathrm{GaN}$ wells in the presence of an electric field, we address the following issues: (i) electronic states in the well in the presence of an electric field; (ii) the excitonic problem in the presence of a field; (iii) a calculation of the absorption spectra and the evaluation of the changes in refractive index of the quantum wells. In our model, we solve the single band effective mass Schrödinger equation for conduction band. The heavy hole and light hole are also solved using single band equations. ${ }^{14}$ The piezoelectric field is included in the potential profile. The exciton problem is addressed by the variational method. We choose the following exciton wave function as the $1 \mathrm{~S}$ state:

$$
\psi_{e x}\left(z_{e}, z_{h}, \rho\right)=N \chi_{e}\left(z_{e}\right) \chi_{h}\left(z_{h}\right) \exp \left(-\frac{\rho}{\lambda}\right),
$$

where $N$ is the normalized constant, $\rho=\left|r_{e}^{\perp}-r_{h}^{\perp}\right|, \chi_{e}, \chi_{h}$ are electron wave function and hole function in the $z$ direction, respectively, and solved through the Schrödinger equations. The parameter $\lambda$ is calculated through the variational principle. $^{15}$

The calculation of absorption spectra has included both exciton and intersubband contribution, where the homoge- 


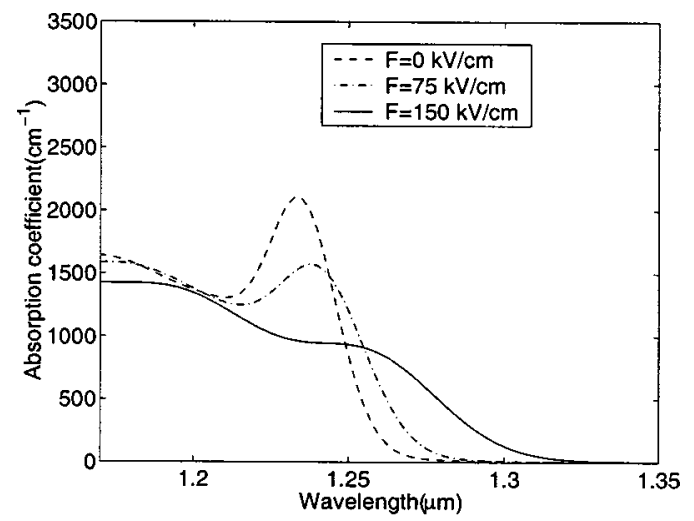

(a)

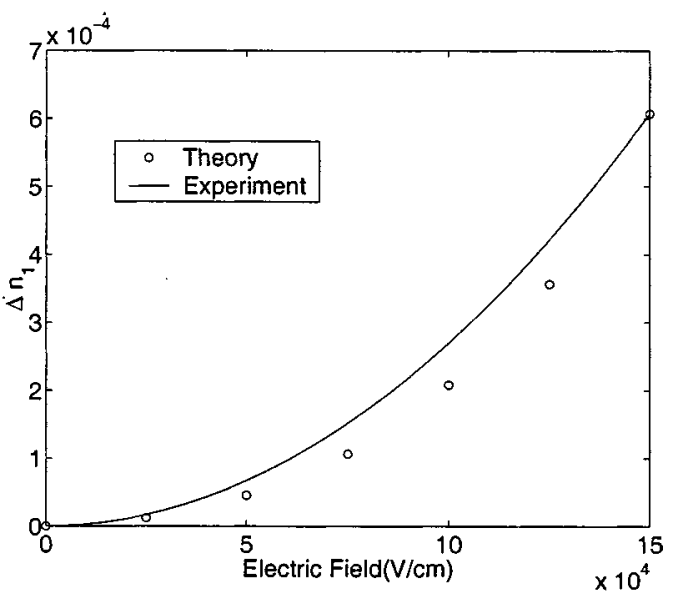

(b)

FIG. 1. (a) Absorption spectra and (b) refractive index changes at $1.33 \mu \mathrm{m}$ for $70 \AA$ InGaAsP/InP quantum wells for TE wave. The optical confinement effect has been included.

neous broadening and well width fluctuation has been taken into account. ${ }^{2}$ The refractive index changes $\delta n$ are calculated through Kramers-Kronig relations from absorption changes $\delta \alpha$ :

$$
\delta n(\omega)=\frac{c}{\pi} P \int_{\omega_{1}}^{\omega_{2}} \frac{\delta \alpha\left(\omega^{\prime}\right) d \omega^{\prime}}{\left(\omega^{\prime}\right)^{2}-\omega^{2}}
$$

To gain confidence in the approach used here, we calculate the electric field induced absorption and refractive-index change in a $1.33 \mu \mathrm{m}$ InGaAsP quantum well modulator with $70 \AA$ quantum wells - a system for which experimental results have been reported. ${ }^{4}$ To fit the measured excitonic linewidth reported in Ref. 4, we assume that the broadening arises from well-width fluctuations of $9 \AA$ rms magnitude and a Lorentzian broadening of $1 \mathrm{meV}$. Two conduction subbands, two heavy hole and two light hole subbands are included in the calculations. The absorption spectra are shown in Fig. 1(a) and agrees with the experimental results. The refractive index changes with field are shown in Fig. 1(b) and are found to agree with experimental results. In our calculations we have assumed that effective waveguide width is

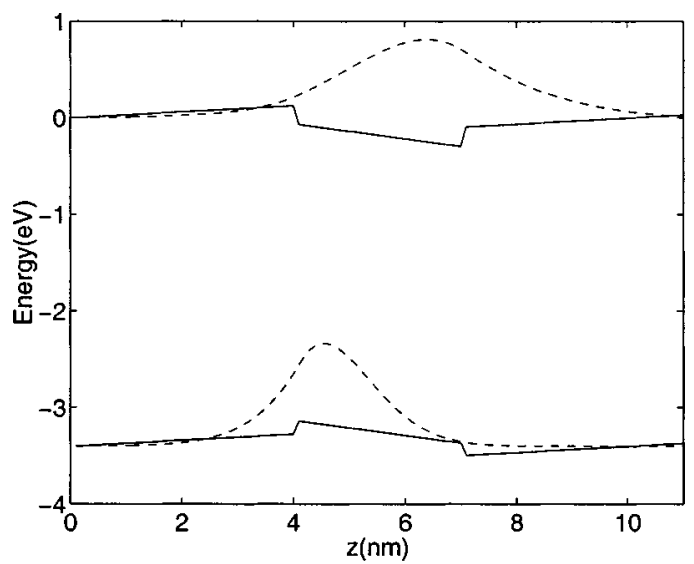

FIG. 2. Conduction band and valence band edges of $\mathrm{GaN} / \mathrm{In}_{0.2} \mathrm{Ga}_{0.8} \mathrm{~N} / \mathrm{GaN}$ quantum wells without bias. The dashed lines represent the ground electronic state and ground hole state, respectively.

$1.2 \mu \mathrm{m}$. These results show that the approach used by us is reasonably accurate and can be relied upon to make predictions for quantum well systems.

The quantum well parameters for the results reported here for InGaN/GaN are chosen to correspond to a system reported in some detail in the literature. For the purpose of choosing the electric field orientation, we assume that growth is in the Ga-faced [0001] direction, which is the case for the usual high quality metalorganic chemical vapor deposition (MOCVD) growth of GaN on sapphire substrate. ${ }^{16}$ The piezoelectric field is then in the negative growth direction. In the multiple quantum well structures, the piezoelectric field is compensated by charges from free charge carriers in the sample and from the ambiance, as is the case for the usual pyroelectric material. ${ }^{17}$ The results here are reported for $30 \AA$ $\mathrm{In}_{0.2} \mathrm{Ga}_{0.8} \mathrm{~N} / \mathrm{GaN}$ quantum wells. The period of the quantum well (well and barrier thickness) is $100 \AA$. The band-gap difference is $0.33 \mathrm{eV}$ and we assume a ratio between conduction band and valence band offset of 60:40. While not unique, this choice gives results consistent with experimental photoluminescence measurement. ${ }^{14}$ The band profiles are plotted in Fig. 2. In the calculations, two subbands are included within the conduction band while eight heavy hole and light hole subbands are included in the valence band. Longitudinal sum rules ${ }^{18}$ are implemented explicitly in order to ensure the Kramers-Kronig transformation accurately generate the refractive index changes. The resulting integrated theoretical absorption is conserved to within $0.2 \%$.

We report results for the case where the effective well width fluctuations are $6 \AA$ and the homogeneous broadening of excitons is chosen $1 \mathrm{meV}$. This gives a broadening around $30 \mathrm{meV}$. Thus the exciton cannot be distinguished from the absorption spectra in Fig. 3(a). This is consistent with experimentally reported results for optical absorption in InGaN/ GaN quantum wells. The electro-optic effect (change in refractive index with applied field) is shown in Fig. 3(b). We find that the effect is quite linear at low fields unlike the case seen in Fig. 1(b). Experimental results ${ }^{11}$ have shown that photoluminescence peak shows a blueshift with the increase of reverse bias. This shift originates from the same QCSE as discussed here. The refractive index change is $1.6 \times 10^{-3}$ at $2.97 \mathrm{eV}$ under electric field $100 \mathrm{kV} / \mathrm{cm}$, which is one mag- 


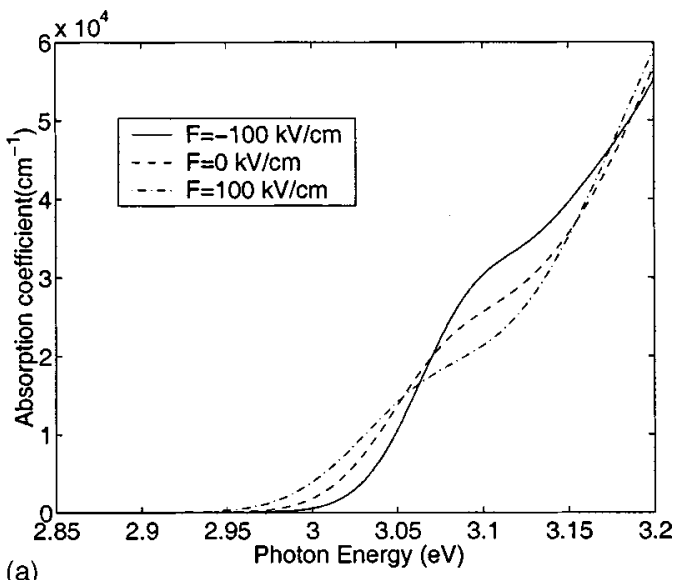

(a)

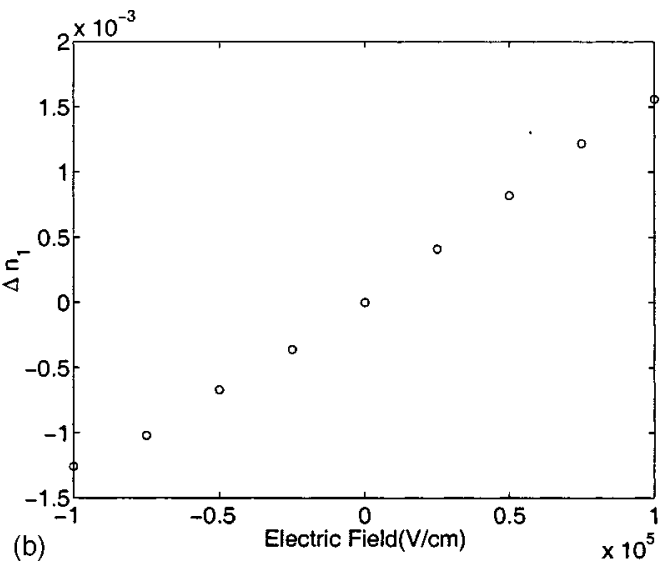

FIG. 3. (a) Absorption spectra and (b) refractive index changes at $2.97 \mathrm{eV}$ for $30 \AA \mathrm{In}_{0.2} \mathrm{Ga}_{0.8} \mathrm{~N} / \mathrm{GaN}$ quantum wells with well width fluctuation $6 \AA$ for TE wave.

nitude larger than the bulk GaN value $1.24 \times 10^{-4}$ at $633 \mathrm{~nm}$ under the same field strength ${ }^{19}$ due to Pockels effect. This value is comparable with $70 \AA \mathrm{InGaAsP} / \mathrm{InP}$ quantum wells at $1.33 \mu \mathrm{m}\left(3.6 \times 10^{-3}\right.$ excluding the optical confinement effect according to our calculation). It is expected that this effect is more pronounced for wider quantum wells. The effective chirp parameter ${ }^{1}$ is 0.70 for this system.

In this letter, the QCSE induced variation in absorption spectra and refractive index change with field has been studied for the InGaN/GaN with built-in piezoelectric field. Our results show that refractive index changes are linear in applied field over a wide range of applied fields.

The authors would like to thank Dr. U. Mishra's research group at UCSB for helpful feedback on this work. This work is funded by a grant from the Office of Naval Research.

${ }^{1}$ J. S. Weiner, D. A. B. Miller, and D. S. Chemla, Appl. Phys. Lett. 50, 842 (1987).

${ }^{2}$ W. Bardyszewski, D. Yevick, Y. Liu, C. Rolland, and S. Bradshaw, J. Appl. Phys. 80, 1136 (1996).

${ }^{3}$ J. E. Zucker, I. Bar-Joseph, B. I. Miller, U. Koren, and D. S. Chemla, Appl. Phys. Lett. 54, 10 (1989).

${ }^{4}$ J. E. Zucker, K. L. Jones, B. I. Miller, and U. Koren, IEEE Photonics Technol. Lett. 2, 32 (1990).

${ }^{5}$ S. Nakamura, M. Senoh, N. Iwasa, and S. Nagahama, Jpn. J. Appl. Phys., Part 2 34, L797 (1995).

${ }^{6}$ S. Nakamura, M. Senoh, S. Nagahama, N. Iwasa, T. Yamada, T. Matsushita, Y. Sugimoto, and H. Kiyoku, Appl. Phys. Lett. 69, 1568 (1996).

${ }^{7}$ F. Bernardini, V. Fiorentini, and D. Vanderbilt, Phys. Rev. B 56, R10024 (1997).

${ }^{8}$ A. D. Bykhovski, V. V. Kaminski, M. S. Shur, Q. C. Chen, and M. A. Khan, Appl. Phys. Lett. 68, 818 (1996).

${ }^{9}$ C. Wetzel, S. Nitta, T. Takeuchi, S. Yamaguchi, H. Amano, and I. Akasaki, MRS Internet J. Nitride Semicond. Res. 3, 31 (1998).

${ }^{10}$ C. Wetzel, T. Takeuchi, H. Amano, and I. Akasaki, J. Appl. Phys. 85, 3786 (1999)

${ }^{11}$ T. Takeuchi, C. Wetzel, S. Yamaguchi, H. Sakai, H. Amano, I. Akasaki, Y. Kaneko, S. Nakagawa, Y. Yamaoka, and N. Yamada, Appl. Phys. Lett. 73, 1691 (1998).

${ }^{12}$ A. Hangleiter, J. S. Im, H. Kollmer, S. Heppel, J. Off, and F. Scholz, MRS Internet J. Nitride Semicond. Res. 3, 15 (1998).

${ }^{13}$ G. Martin, A. Botchkarev, A. Rockett, and H. Morkoç, Appl. Phys. Lett. 68, 2541 (1996).

${ }^{14}$ H. Jiang, M. Minsky, S. Keller, E. Hu, J. Singh, and S. P. DenBaars IEEE J. Quantum Electron. (to be published).

${ }^{15}$ R. L. Greene and K. K. Bajaj, Solid State Commun. 45, 831 (1983).

${ }^{16}$ F. A. Ponce, D. P. Bour, W. T. Young, M. Saunders, and J. W. Steeds, Appl. Phys. Lett. 69, 337 (1996).

${ }^{17}$ A. D. Bykhovski, V. V. Kaminski, M. S. Shur, Q. C. Chen, and M. A. Khan, Appl. Phys. Lett. 69, 3254 (1996).

${ }^{18}$ D. A. B. Miller, J. S. Weiner, and D. S. Chemla, IEEE J. Quantum Electron. 22, 1816 (1986).

${ }^{19}$ X.-C. Long, R. A. Myers, S. R. J. Brueck, R. Ramer, K. Zheng, and S. D. Hersee, Appl. Phys. Lett. 67, 1349 (1995). 